\title{
Ethnologies
}

Le réseau du Canada : Étude du mode migratoire de la France vers la Nouvelle-France,1628-1662. Gervais Carpin (Québec, Septentrion et Presses de l'Université de Paris-Sorbonne, 2001, 552 p., ISBN 2-89448-197-7)

De Français à paysans : Modernité et tradition dans le peuplement du Canada français. Leslie Choquette (Québec, Septentrion et Presses de l'Université de Paris-Sorbonne, 2001, 323 p., ISBN 2-89448-196-9)

\section{Anne-Hélène Kerbiriou}

Volume 24, numéro 1, 2002

Espace

Space

URI : https://id.erudit.org/iderudit/006549ar

DOI : https://doi.org/10.7202/006549ar

Aller au sommaire du numéro

Éditeur(s)

Association Canadienne d'Ethnologie et de Folklore

ISSN

1481-5974 (imprimé)

1708-0401 (numérique)

Découvrir la revue

Citer ce compte rendu

Kerbiriou, A.-H. (2002). Compte rendu de [Le réseau du Canada : Étude du mode migratoire de la France vers la Nouvelle-France,1628-1662. Gervais Carpin (Québec, Septentrion et Presses de l'Université de Paris-Sorbonne, 2001, 552 p., ISBN 2-89448-197-7) / De Français à paysans : Modernité et tradition dans le peuplement du Canada français. Leslie Choquette (Québec, Septentrion et Presses de l'Université de Paris-Sorbonne, 2001, 323 p., ISBN 2-89448-196-9)]. Ethnologies, 24(1), 315-319. https://doi.org/10.7202/006549ar d'utilisation que vous pouvez consulter en ligne. 
Le réseau du Canada : Étude du mode migratoire de la France vers la Nouvelle-France, 1628-1662. Gervais Carpin (Québec, Septentrion et Presses de I'Université de Paris-Sorbonne, 2001, 552 p., ISBN 2-89448197-7)

De Français à paysans : Modernité et tradition dans le peuplement du Canada français. Leslie Choquette (Québec, Septentrion et Presses de I'Université de Paris-Sorbonne, 2001, 323 p., ISBN 2-89448-196-9)

L'ouvrage de Leslie Choquette, traduit par Gervais Carpin, avait été édité en 1997 sous le titre Frenchmen into Peasants. Le choix des Éditions du Septentrion de le rééditer en français, conjointement à l'ouvrage de Gervais Carpin, nous paraît être une initiative intéressante, car les deux sont heureusement complémentaires. Avec Le réseau du Canada, Gervais Carpin se penche sur l'immigration en Nouvelle-France à un niveau global, considérant avec la même minutie les immigrants occasionnels, les " engagés ", que ceux qui y ont laissé une descendance, ce en quoi son étude est novatrice. Leslie Choquette livre une étude allant d'une certaine manière dans le même sens, puisqu'elle aussi dépasse «le cadre des seuls pionniers » pour aborder l'ensemble des migrants partis de France vers le Canada. Mais son étude prolonge celle de Gervais Carpin, puisqu'elle s'intéresse au sort de ceux qui sont restés dans la colonie, dans leur majorité des artisans qui se sont «attachés à la terre» et qui ont fondé une société rurale «traditionnelle», moins par leur volonté propre que parce que l'évolution économique générale des siècles suivants les y a contraints.

Il y avait 3000 Français installés en Nouvelle-France en 1662 contre environ 40000 autres Européens en Nouvelle-Angleterre. L'objectif de Gervais Carpin est de «trouver le moyen d'analyser en lui-même le mode migratoire de la France vers la Nouvelle-France afin de faire surgir l'explication de ce faible flux migratoire » (345). Il concède qu'hormis une série de documents notariés son étude ne se fonde guère sur des documents inédits. Mais il les aborde d'un oeil neuf, les mettant en relation dans leur ensemble, y cherchant les liens, serrés ou diffus, de relations interpersonnelles, afin de faire apparaître un mode migratoire propre au contexte de la Nouvelle-France. Le moindre de ses mérites n'est pas de faire du neuf avec du vieux, et de parvenir à faire ressortir des éléments passés jusque là inaperçus dans ces archives déjà largement étudiées. Il nous livre une étude incroyablement minutieuse et fouillée au bout de laquelle, chose rare, on en arrive quasiment à percevoir et ressentir les motivations de personnes modestes et réelles, dégagées des 
abstractions des grands contextes historiques. À force de minutie, l'historien devient ethnologue, et cherche la réponse à la question récurrente dans les travaux précédents: "pourquoi le peuplement de la colonie eut-il le rythme qui fut le sien et que certains appellent échec \$(22) ?

Tenue en général pour responsable de cet échec, la Compagnie de la Nouvelle-France est l'objet détaillé de la première partie de l'ouvrage. Le mandat de la Compagnie lui imposait de «faire passer» 4000 hommes ou femmes en quinze ans. Mais « il n'était pas écrit d'établir, de peupler» (81). Ce mandat a été largement rempli par le passage de 5000 personnes dans le temps alloué. L'analyse détaillée de la Compagnie, véritable "créature " de Richelieu, son fonctionnement, son évolution, son organisation en liaisons de personne à personne, fait bien apparaître que l'échec, si c'en est un, n'est pas attribuable à la Compagnie elle-même, mais plutôt à certaines malchances et aux manquements de son "créateur ». Car les Associés eux-mêmes, individus mis en lumière au travers de documents observés au microscope, atteignent à une "réalité » rarement perçue ; ce sont des personnes aux motivations pécuniaires indéniables, mais qui ont persisté dans des investissements considérables tant que faire se pouvait, et dont on peut ressentir, au-delà des opérations financières, l'enthousiasme pour ce qui n'était encore qu'une idée, la Nouvelle-France.

La deuxième partie de l'ouvrage, le corps du livre, porte sur l'aspect organisationnel du réseau et les recruteurs. Après avoir passé en revue la période des premiers embarquements, de 1628 à 1632, l'auteur détaille la succession des organismes recruteurs, la Compagnie de la NouvelleFrance, puis la Communauté des habitants, les recruteurs privés pour la vallée du Saint-Laurent et enfin les seigneurs recruteurs et les communautés de traite de l'Atlantique. Il apparaît clairement que, pour la Compagnie de la Nouvelle-France, le fonctionnement des embarquements et le recrutement reposait sur « un nombre très limité d'associés et toujours les mêmes, parmi lesquels... Antoine Cheffault et Jean Rozée »(164). Par la suite, le recrutement opéré par la Communauté des habitants opère en fonction de liens d'affaires et de liens familiaux auxquels on pouvait s'attendre étant donnée «la propension de cette époque aux alliances matrimoniales endogames à l'intérieur des groupes professionnels et sociaux » (193). Les recruteurs privés sont principalement les communautés religieuses, mais contrairement aux idées communément admises, elles n'apparaissent 
pas comme les agents les plus actifs du recrutement. Mais elles aussi fonctionnaient en réseau, en témoigne un schéma fort intéressant sur la «Représentation des liens connus entre les 8 familles d'émigrants autonomes de Marans en 1659 »(230), illustrant aussi bien l'enchevêtrement des liens de parenté que la minutie du chercheur. C'est un exemple du volume de renseignements que contient cet ouvrage et qui en fait, sinon une somme définitive, du moins une base extrêmement solide pour d'autres travaux, notamment par le nombre et la qualité de ses annexes, telle que la liste des engagés de la Rochelle entre 1633 et 1662 par exemple (456-499).

C'est à l'examen des autres réseaux de recrutement que l'on se rend compte de la prépondérance de la Compagnie de la Nouvelle-France. C'était un appareil issu d'une décision politique de Richelieu et qui avait pour fonction "d'être l'outil de cette politique ». Richelieu avait cru pouvoir insuffler le peuplement colonial par le seul financement privé. Or, après 1662, avec le passage du pouvoir à Colbert, qui instaure une nouvelle politique, le peuplement de la colonie connut un élan inégalé, passant de 3000 à 6000 habitants en trois ans. "Cela ne dura pas... Mais ces quelques années suffisent à montrer que, faute d'un Eldorado basé sur les cultures du tabac ou du sucre ou faute d'un élan utopique missionnaire, seul un financement public soutenant un projet à long terme pouvait donner des résultats convaincants » (350).

La troisième partie de l'ouvrage de Gervais Carpin porte sur l'objet du réseau, les recrutés. À partir d'une analyse de 529 contrats d'engagements, il démontre que plus de la moitié des immigrants, au début de la colonie, provenaient du bassin de La Rochelle, ville qui se trouvait « en situation particulière d'attraction migratoire suite au repeuplement très important qui lui était nécessaire ». Les migrants qui traversaient l'Atlantique étaient déjà au préalable des gens qui se déplaçaient à l'intérieur de la France. Ils étaient plus nombreux à venir des campagnes que des villes, mais étant donné que la France rurale était quatre fois plus peuplée que la France urbaine, l'ensemble de la population passant outre-Atlantique se présente comme largement plus urbaine et artisanale que la population de l'hexagone (296).

Cette idée fonde l'ouvrage de Leslie Choquette, qui étudie l'origine régionale et sociale des migrants, en établissant tout d'abord la difficulté qui se présente à vouloir dessiner les contours d'une population modeste et mouvante. Les migrations faisaient partie de la vie des Français de 
l'Ancien Régime, et partir outre-Atlantique, dans ce contexte, n'est pas seulement une grande aventure, mais aussi le prolongement, sur une plus grande distance, de pratiques usuelles (188). L'ensemble de l'ouvrage entreprend donc un portrait fouillé de la population migrante. S'il n'est pas de lecture légère, il est néanmoins fort instructif. Pour Leslie Choquette, les migrants vers la Nouvelle-France appartiennent à une "géographie de la modernité ». Ce sont souvent de jeunes adultes (moins de trente ans) de la France du Nord-Ouest, mobiles, pas encore insérés dans leur milieu, relevant d'une économie atlantique, qui comptent dans leurs rangs « très peu de paysans, un fort contingent des membres de l'élite... un nombre formidable d'artisans » (110), des femmes, quelques juifs et protestants discrets; la plupart sont catholiques, mais bien moins dévots qu'on ne le penserait à l'ère de la Contre-Réforme (chap. V).

Leslie Choquette fait remarquer que, en un temps où $80 \%$ des gens gagnaient leur vie directement à partir de la terre, les immigrants vers la Nouvelle-France, majoritairement d'origine urbaine, ou artisans en milieu rural, n'étaient pas représentatifs de leur communautés d'origine. Elle fait également remarquer au chapitre VI, à la suite d'autres auteurs, l'absence totale de liens entre le mouvement migratoire au Canada et les fluctuations de l'économie agricole. Son travail aborde donc, dès les premières pages, le «paradoxe d'une nation profondément rurale se reproduisant outre-mer à partir de ses éléments les moins ruraux » (33).

Une telle question justifie à elle-seule l'intérêt que l'on peut porter à cet ouvrage. Mais il faut le développement de tout le livre, une approche au plus près, et finement menée, des caractéristiques d'une population, pour y voir se développer la réponse, en conclusion. Du même coup, elle répond également, en une large synthèse, à l'interrogation de l'ouvrage précédent, à savoir l'inégalité, en termes de peuplement, de la population canadienne-française et de la population américaine. Gervais Carpin a démontré de quelle manière la faiblesse du peuplement relevait à l'origine à la fois de la politique de Richelieu et des fluctuations des investissements privés. Leslie Choquette prolonge cette réponse en montrant en quoi l'État français a freiné les initiatives de développement économique du Canada, puisque, selon une lettre royale, « tout ce qui pourrait faire concurrence avec les manufactures du royaume ne doit jamais être fait dans les colonies »(242). Sans possibilité d'exportations, les colons français se sont transformés en agriculteurs, « renonçant à produire au-delà de leurs besoins » (243). 
La phrase finale de l'ouvrage de Leslie Choquette revient sur la question de la différence quantitative du peuplement entre les Amériques française et britannique et ouvre d'autres perspectives : «les modèles de mobilité, de vie économique, de reproduction familiale, de structures sociales et même de culture avaient beaucoup en commun, et se comprennent peut-être mieux comme faisant partie d'une expérience coloniale atlantique pourtant fortement diversifiée »(261).

La lecture conjointe de ces deux ouvrages nous livre un portrait fouillé de la genèse d'une population, et il s'en dégage une direction générale qui laisse pressentir d'autres recherches. Tout d'abord, le fait d'avoir établi clairement que cette population soit en grande partie composée d'artisans, type d'individus que Leslie Choquette qualifie de «modernes » bien que la transmission de leurs métiers, au XVIIe siècle, relève encore clairement de pratiques anciennes, renouvelle la pertinence des travaux des ethnologues sur la culture matérielle traditionnelle du Québec, et peut permettre de nouvelles interrogations sur l'origine géographique et la pérennité de certaines pratiques. Et, enfin, notre perception du peuplement du Canada français se trouve renouvelée. La fondation de la Nouvelle-France, en définitive, et contrairement à nombre d'idées reçues, paraît moins le résultat de la politique de Richelieu que de l'initiative d'individus peu nombreux, mais volontaires et déterminés, allant d'eux-mêmes au-devant d'une vie qu'ils espéraient plus libre. Contrairement à leurs espérances, leurs descendants se sont trouvés «enfermés " dans un mode de vie rural qu'ils ne pouvaient prévoir, en raison des pesanteurs de cet Ancien Régime auxquelles ils souhaitaient échapper. La société rurale traditionnelle au Québec est en fait la résultante de l'adaptation d'une population non-rurale à des contraintes imprévisibles et de l'isolement progressif de gens qui, au départ, étaient liés entre eux. Ces deux faits réunis peuvent ouvrir la voie à d'autres recherches de grande ampleur et seraient susceptibles de renouveler totalement notre vision du peuplement du Canada français. 